\title{
Generalization of Singh's Common Fixed Point Theorem
}

\author{
J.Jeyachristy Priskillal, G.Sheeba Merlin
}

\begin{abstract}
In this article, I generalize Singh's common fixed point theorem for compatible mappings in fuzzy metric spaces. Examples are given to support the results proved herein.

Index Terms - fuzzy metric space; compatible mapping; common fixed point.
\end{abstract}

\section{INTRODUCTION}

The author defined intuitionistic $(\psi, \eta)$ contractive mapping in [6]. Using the definition of $\psi$, we gave a common fixed point theorem. The generalization of the commuting mapping concept is compatible mapping which is introduced by Gerald Jungck[3]. This concept was generalized to fuzzy metric spaces by Mishra et al.[7]. Vasuki[13] proved a fuzzier version of the result of Pant[8]. She proved a common fixed point theorem using R-weakly commuting. Further, some Mathematicians proved common fixed point theorem for compatible mappings[11],[12],[10]. In the year 2000, Singh[1] proved a common fixed point result for two compatible pairs of maps in fuzzy metric spaces as follows:

Let A,B,S and T be self-mappings of a complete fuzzy metric space $(X, M, *)$ with $a * b=\min (a, b)$ satisfy the following conditions:

(I) $\mathrm{BX} \subset \mathrm{SX}, \mathrm{AX} \subset \mathrm{TX}$,

(II) A,S and B,T are compatible,

(III) $\mathrm{S}$ and $\mathrm{T}$ are continuous,

(IV) $\mathrm{M}(\mathrm{Au}, \mathrm{Bv}, \mathrm{kt}) \geq \min \{\mathrm{M}(\mathrm{Su}, \mathrm{Tv}, \mathrm{t}), \mathrm{M}(\mathrm{Au}, \mathrm{Su}, \mathrm{t})$,

$\mathrm{M}(\mathrm{Bv}, \mathrm{Tv}, \mathrm{t}), \mathrm{M}(\mathrm{Su}, \mathrm{Bv}, 2 \mathrm{t}), \mathrm{M}(\mathrm{Au}, \mathrm{Tv}, \mathrm{t})\}$, for all $\mathrm{u}, \mathrm{v} \in \mathrm{X}, \mathrm{t}$ $>0$ and $\mathrm{k} \in(0,1)$.

Then A,B,S and T have a unique common fixed point.

In our paper[6], $\psi$ is defined as follows,

Let $\Psi$ be the class of all mappings $\psi:[0,1] \rightarrow[0,1]$ such that

(i) $\psi$ is non-decreasing and $\lim _{n \rightarrow \infty} \psi^{\mathrm{n}}(\mathrm{s})=1, \forall \mathrm{s} \in(0,1]$;

(ii) $\psi(\mathrm{s})>\mathrm{s}, \forall \mathrm{s} \in(0,1)$;

(iii) $\psi(1)=1$;

Example 1.1. [6] Define $\psi:[0,1] \rightarrow[0,1]$ by

$\psi(\mathrm{s})=\frac{2 s}{s+1}, \forall \mathrm{s} \in[0,1]$.

$\psi^{2}(\mathrm{~s})=\frac{4 s}{3 s+1}, \psi^{3}(\mathrm{~s})=\frac{8 s}{7 s+1}, \ldots$,

$\psi^{\mathrm{n}}(\mathrm{s})=\frac{2^{n} s}{\left(2^{n}-1\right) s+1}, \forall \mathrm{s} \in[0,1]$.

Revised Manuscript Received on April 12, 2019.

Dr. J.Jeyachristy Priskillal, School of Maritime Studies, Vels Institute of Technology, Sciences and Advanced Studies,Chennai-600127, T.N, India

Dr. G.Sheeba Merlin, Department of Mathematics, Karunya Institute of Technology and Sciences, Coimbatore, T.N, India. $\lim _{n \rightarrow \infty} \psi^{\mathrm{n}}(\mathrm{s})=\lim _{n \rightarrow \infty} \frac{2^{n} s}{\left(2^{n}-1\right) s+1}=1, \forall \mathrm{s} \in(0,1]$.

Clearly, $\psi(\mathrm{s})>\mathrm{s}, \forall \mathrm{s} \in(0,1)$ and $\psi(1)=1$.

\section{PRELIMINARIES}

Definition 2.1. [9] A binary operation $*:[0,1] \times[0,1] \rightarrow$ $[0,1]$ is called t-norm if the following conditions hold:

(a) $*$ is associative and commutative;

(b) a * $1=\mathrm{a}, \forall \mathrm{a} \in[0,1]$;

(c) a $*$ b $\leq \mathrm{c} * \mathrm{~d}$ whenever $\mathrm{a} \leq \mathrm{c}$ and $\mathrm{b} \leq \mathrm{d}, \forall \mathrm{a}, \mathrm{b}, \mathrm{c}, \mathrm{d} \in$ $[0,1]$.

If $*$ is continuous then it is called a continuous t-norm.

Definition 2.2.[5] Let $\mathrm{X}$ be an arbitrary set, $*$ be a continuous t-norm, and $M$ be fuzzy sets on $X^{2} \times(0, \infty)$. Consider the following conditions $\forall \mathrm{u}, \mathrm{v}, \mathrm{w} \in \mathrm{X}$ and $\mathrm{t}>0$,

(i) $\mathrm{M}(\mathrm{u}, \mathrm{v}, 0)=0$;

(ii) $\mathrm{M}(\mathrm{u}, \mathrm{v}, \mathrm{t})=1$ if and only if $\mathrm{u}=\mathrm{v}$;

(iii) $\mathrm{M}(\mathrm{u}, \mathrm{v}, \mathrm{t})=\mathrm{M}(\mathrm{v}, \mathrm{u}, \mathrm{t})$;

(iv) $\mathrm{M}(\mathrm{u}, \mathrm{w}, \mathrm{t}+\mathrm{s}) \geq \mathrm{M}(\mathrm{u}, \mathrm{v}, \mathrm{t}) * \mathrm{M}(\mathrm{v}, \mathrm{w}, \mathrm{s})$;

(v) $\mathrm{M}(\mathrm{u}, \mathrm{v}, \mathrm{t}):(0, \infty) \rightarrow[0,1]$ is left continuous;

The pair $(\mathrm{M}, *)$ is called fuzzy metric on $\mathrm{X}$. The triple $(\mathrm{X}, \mathrm{M}, *)$ is called a fuzzy metric space.

Example 2.3. [2] Let $(\mathrm{X}, \mathrm{d})$ be a metric space. Denote $\mathrm{a} * \mathrm{~b}=\mathrm{ab}, \forall \mathrm{a}, \mathrm{b} \in[0,1]$ and let $\mathrm{M}_{\mathrm{d}}$ be fuzzy set on $\mathrm{X} \times \mathrm{X} \times(0,+\infty)$ defined as follows:

$\mathrm{M}_{\mathrm{d}}(\mathrm{u}, \mathrm{v}, \mathrm{t})=\frac{t}{t+d(u, v)}, \forall \mathrm{t}>0$, then $\left(\mathrm{X}, \mathrm{M}_{\mathrm{d}}, *\right)$ is a fuzzy metric space.

Definition 2.4. [4] Let (X,M,*) be a fuzzy metric space. A sequence $\left\{u_{n}\right\}$ in $X$ is called

(a) convergent to a point $u \in X$ if and only if $\lim _{n \rightarrow \infty}$ $\mathrm{M}\left(\mathrm{u}_{\mathrm{n}}, \mathrm{u}, \mathrm{t}\right)=1, \forall \mathrm{t}>0$,

(b) Cauchy if $\lim _{n \rightarrow \infty} \mathrm{M}\left(\mathrm{u}_{\mathrm{n}}, \mathrm{u}_{\mathrm{n}+\mathrm{p}}, \mathrm{t}\right)=1, \forall \mathrm{t}>0$ and $\mathrm{p}>0$.

Definition 2.5. [4] A fuzzy metric space is said to be complete if every Cauchy sequence in $\mathrm{X}$ is convergent.

Definition 2.6. [7] In a fuzzy metric space (X,M,*), two self mappings $\mathrm{A}$ and $\mathrm{B}$ are said to be compatible if $\lim _{n \rightarrow \infty}$ $\mathrm{M}\left(\mathrm{ABu}_{\mathrm{n}}, \mathrm{BAu}_{\mathrm{n}}, \mathrm{t}\right)=1$ whenever $\mathrm{u}_{\mathrm{n}}$ is a sequence in $\mathrm{X}$ such that $\lim _{n \rightarrow \infty} A u_{n}=\lim _{n \rightarrow \infty} B u_{n}=w$ for some $\mathrm{w} \in X$.

Lemma 2.7. [8] If $\mathrm{A}$ and $\mathrm{B}$ are compatible mappings on a fuzzy metric space $\mathrm{X}$ and $\mathrm{Au}_{\mathrm{n}}, \mathrm{Bu}_{\mathrm{n}} \rightarrow \mathrm{w}$ for some $\mathrm{w}$ in $\mathrm{X}\left(\mathrm{u}_{\mathrm{n}}\right.$ being a sequence in $\mathrm{X}$ ) then $\mathrm{ABu}_{\mathrm{n}} \rightarrow \mathrm{Bw}$ provided $\mathrm{B}$ is continuous (at w)

Published By:

Blue Eyes Intelligence Engineering 


\section{GENERALIZATION OF SINGH'S COMMON FIXED POINT THEOREM}

\section{MAIN RESULTS}

Proposition 3.1. Let A and B be compatible mappings of a fuzzy metric space $(\mathrm{X}, \mathrm{M}, *)$ into itself. If $\mathrm{Aw}=\mathrm{Bw}$ for some

$\mathrm{w} \in \mathrm{X}$, then $\mathrm{ABw}=\mathrm{BAw}$.

Proof. Suppose that $\left\{\mathrm{u}_{\mathrm{n}}\right\}$ is a sequence in $\mathrm{X}$ defined by $\mathrm{u}_{\mathrm{n}}=\mathrm{w}_{\mathrm{n}}=1,2, \ldots$ for some $\mathrm{w} \in \mathrm{X}$ and $\mathrm{Aw}=\mathrm{Bw}$.

Then we have $\mathrm{Au}_{\mathrm{n}}, \mathrm{Bu}_{\mathrm{n}} \rightarrow \mathrm{Aw}$ as $\mathrm{n} \rightarrow \infty$.

Since $\mathrm{A}$ and $\mathrm{B}$ are compatible mapping,

$\mathrm{M}(\mathrm{ABw}, \mathrm{BAw}, \mathrm{t})=\lim _{n \rightarrow \infty} \mathrm{M}\left(\mathrm{ABu}_{\mathrm{n}}, \mathrm{BAu}, \mathrm{t}\right)=1$.

Hence, we have $\mathrm{ABw}=\mathrm{BAw}$.

Since $A w=B w$, we have $A B w=B A w$.

Theorem 3.2. Let $\mathrm{A}, \mathrm{B}, \mathrm{S}$ and $\mathrm{T}$ be self-mappings of a complete fuzzy metric space $(\mathrm{X}, \mathrm{M}, *)$ with $\mathrm{a} * \mathrm{~b}=\min (\mathrm{a}, \mathrm{b})$ satisfy the following conditions:

(I) $\quad \mathrm{BX} \subset \mathrm{SX}, \mathrm{AX} \subset \mathrm{TX}$,

(II) $\mathrm{A}, \mathrm{S}$ and $\mathrm{B}, \mathrm{T}$ are compatible,

(III) One of $\mathrm{A}, \mathrm{B}, \mathrm{S}$ and $\mathrm{T}$ is continuous,

(IV) $\mathrm{M}(\mathrm{Au}, \mathrm{Bv}, \mathrm{t}) \geq \psi[\min \{\mathrm{M}(\mathrm{Su}, \mathrm{Tv}, \mathrm{t}), \mathrm{M}(\mathrm{Au}, \mathrm{Su}, \mathrm{t})$, $\mathrm{M}(\mathrm{Bv}, \mathrm{Tv}, \mathrm{t})$,

$\mathrm{M}(\mathrm{Su}, \mathrm{Bv}, 2 \mathrm{t}), \mathrm{M}(\mathrm{Au}, \mathrm{Tv}, \mathrm{t})\}]$,

for all $u, v \in X$ and $t>0$.

Then A,B,S and T have a unique common fixed point.

Proof. Consider a point $\mathrm{u}_{0} \in \mathrm{X}$.

Since BX $\subset \mathrm{SX}$ and $\mathrm{AX} \subset \mathrm{TX}$, We can define a sequence $\left\{\mathrm{v}_{\mathrm{n}}\right\}$ in $\mathrm{X}$ as follows:

there exists $u_{1} \in X$ such that $\mathrm{Au}_{0}=\mathrm{Tu}_{1}=\mathrm{v}_{0}$.

there exists $\mathrm{u}_{2} \in \mathrm{X}$ such that $\mathrm{Bu}_{1}=\mathrm{Su}_{2}=\mathrm{v}_{1}$.

there exists $u_{2 n+1} \in X$ such that $\mathrm{Au}_{2 \mathrm{n}}=\mathrm{Tu}_{2 \mathrm{n}+1}=\mathrm{v}_{2 \mathrm{n}}$.

there exists $u_{2 n+2} \in X$ such that $\mathrm{Bu}_{2 n+1}=\mathrm{Su}_{2 n+2}=v_{2 n+1}$.

Now, for all $\mathrm{t}>0$,

$\mathrm{M}\left(\mathrm{v}_{2 \mathrm{n}}, \mathrm{v}_{2 \mathrm{n}+1}, \mathrm{t}\right)=\mathrm{M}\left(\mathrm{Au}_{2 \mathrm{n}}, \mathrm{Bu}_{2 \mathrm{n}+1}\right)$

$\geq \psi\left[\min \left\{\mathrm{M}\left(\mathrm{Su}_{2 \mathrm{n}}, \mathrm{Tu}_{2 \mathrm{n}+1}, \mathrm{t}\right), \mathrm{M}\left(\mathrm{Au}_{2 \mathrm{n}}, \mathrm{Su}_{2 \mathrm{n}}, \mathrm{t}\right)\right.\right.$,

$\mathrm{M}\left(\mathrm{Bu}_{2 \mathrm{n}+1}, \mathrm{Tu}_{2 \mathrm{n}+1}, \mathrm{t}\right), \mathrm{M}\left(\mathrm{Su}_{2 \mathrm{n}}, \mathrm{Bu}_{2 \mathrm{n}+1}, 2 \mathrm{t}\right)$,

$\left.\left.\mathrm{M}\left(\mathrm{Au}_{2 \mathrm{n}}, \mathrm{Tu}_{2 \mathrm{n}+1}, \mathrm{t}\right)\right\}\right]$

$=\psi\left[\min \left\{\mathrm{M}\left(\mathrm{v}_{2 \mathrm{n}-1}, \mathrm{v}_{2 \mathrm{n}}, \mathrm{t}\right), \mathrm{M}\left(\mathrm{v}_{2 \mathrm{n}}, \mathrm{v}_{2 \mathrm{n}-1}, \mathrm{t}\right)\right.\right.$,

$\mathrm{M}\left(\mathrm{v}_{2 \mathrm{n}+1}, \mathrm{v}_{2 \mathrm{n}}, \mathrm{t}\right), \mathrm{M}\left(\mathrm{v}_{2 \mathrm{n}-1}, \mathrm{v}_{2 \mathrm{n}+1}, 2 \mathrm{t}\right)$,

$\left.\left.\mathrm{M}\left(\mathrm{v}_{2 \mathrm{n}}, \mathrm{v}_{2 \mathrm{n}}, \mathrm{t}\right)\right\}\right]$

$=\psi\left[\min \left\{\mathrm{M}\left(\mathrm{v}_{2 \mathrm{n}-1}, \mathrm{v}_{2 \mathrm{n}}, \mathrm{t}\right), \mathrm{M}\left(\mathrm{v}_{2 \mathrm{n}+1}, \mathrm{v}_{2 \mathrm{n}}, \mathrm{t}\right)\right.\right.$,

$\left.\left.\left[\mathrm{M}\left(\mathrm{v}_{2 \mathrm{n}-1}, \mathrm{v}_{2 \mathrm{n}}, \mathrm{t}\right) * \mathrm{M}\left(\mathrm{v}_{2 \mathrm{n}}, \mathrm{v}_{2 \mathrm{n}+1}, \mathrm{t}\right)\right], 1\right\}\right]$

$=\psi\left[\mathrm{M}\left(\mathrm{v}_{2 \mathrm{n}-1}, \mathrm{v}_{2 \mathrm{n}}, \mathrm{t}\right), \mathrm{M}\left(\mathrm{v}_{2 \mathrm{n}}, \mathrm{v}_{2 \mathrm{n}+1}, \mathrm{t}\right)\right]$

$=\psi\left[\min \left\{\mathrm{M}\left(\mathrm{v}_{2 \mathrm{n}-1}, \mathrm{v}_{2 \mathrm{n}}, \mathrm{t}\right), \mathrm{M}\left(\mathrm{v}_{2 \mathrm{n}}, \mathrm{v}_{2 \mathrm{n}+1}, \mathrm{t}\right)\right\}\right]$.

Since $\psi(\mathrm{s})>\mathrm{s}$, for all $\mathrm{s} \in(0,1)$,

$\mathrm{M}\left(\mathrm{v}_{2 \mathrm{n}}, \mathrm{v}_{2 \mathrm{n}+1}, \mathrm{t}\right) \geq \psi\left(\mathrm{M}\left(\mathrm{v}_{2 \mathrm{n}}, \mathrm{v}_{2 \mathrm{n}+1}, \mathrm{t}\right)\right)>\mathrm{M}\left(\mathrm{v}_{2 \mathrm{n}}, \mathrm{v}_{2 \mathrm{n}+1}, \mathrm{t}\right)$ which is a contradiction.

Therefore, $\mathrm{M}\left(\mathrm{v}_{2 \mathrm{n}}, \mathrm{v}_{2 \mathrm{n}}+1, \mathrm{t}\right) \geq \psi\left(\mathrm{M}\left(\mathrm{v}_{2 \mathrm{n}-1}, \mathrm{v}_{2 \mathrm{n}}, \mathrm{t}\right)\right)$.

That is, $\quad M\left(v_{n}, v_{n+1}, t\right) \geq \psi\left(M\left(v_{n-1}, v_{n}, t\right)\right)$.

Continuing this process, we can get

$\mathrm{M}\left(\mathrm{v}_{\mathrm{n}}, \mathrm{v}_{\mathrm{n}+1}, \mathrm{t}\right) \geq \psi\left(\mathrm{M}\left(\mathrm{v}_{\mathrm{n}-1}, \mathrm{v}_{\mathrm{n}}, \mathrm{t}\right)\right) \geq \psi^{2}\left(\mathrm{M}\left(\mathrm{v}_{\mathrm{n}-2}, \mathrm{v}_{\mathrm{n}-1}, \mathrm{t}\right)\right) \geq \cdots$ $\geq \psi^{\mathrm{n}}\left(\mathrm{M}\left(\mathrm{v}_{1}, \mathrm{v}_{0}, \mathrm{t}\right)\right)$.

That is, $\mathrm{M}\left(\mathrm{v}_{\mathrm{n}}, \mathrm{v}_{\mathrm{n}+1}, \mathrm{t}\right) \geq \psi^{\mathrm{n}}\left(\mathrm{M}\left(\mathrm{v}_{1}, \mathrm{v}_{0}, \mathrm{t}\right)\right)$.

Taking limit as $\mathrm{n} \rightarrow \infty$ and $\lim _{n \rightarrow \infty} \psi^{\mathrm{n}}(\mathrm{s})=1$, for all $\mathrm{s} \in$ $(0,1]$,

$\lim _{n \rightarrow \infty} \mathrm{M}\left(\mathrm{v}_{\mathrm{n}}, \mathrm{v}_{\mathrm{n}+1}, \mathrm{t}\right)=1$.

Similarly, we can prove

$\mathrm{M}\left(\mathrm{v}_{\mathrm{n}+1}, \mathrm{v}_{\mathrm{n}+2}, \mathrm{t}\right) \geq \psi^{\mathrm{n}}\left(\mathrm{M}\left(\mathrm{v}_{2}, \mathrm{v}_{1}, \mathrm{t}\right)\right)$.

$\lim _{n \rightarrow \infty} \mathrm{M}\left(\mathrm{v}_{\mathrm{n}+1}, \mathrm{v}_{\mathrm{n}+2}, \mathrm{t}\right)=1$.
Now for all $\mathrm{p}>0$,

$\mathrm{M}\left(\mathrm{v}_{\mathrm{n}}, \mathrm{v}_{\mathrm{n}+\mathrm{p}}, \mathrm{t}\right) \geq \mathrm{M}\left(\mathrm{v}_{\mathrm{n}}, \mathrm{v}_{\mathrm{n}+1}, \frac{t}{p}\right) * \ldots * \mathrm{M}\left(\mathrm{v}_{\mathrm{n}+\mathrm{p}-1}, \mathrm{v}_{\mathrm{n}+\mathrm{p}}, \frac{t}{p}\right)$.

Taking limit $\mathrm{n} \rightarrow \infty$, we have,

$$
\begin{aligned}
\lim _{n \rightarrow \infty} \mathrm{M}\left(\mathrm{v}_{\mathrm{n}}, \mathrm{v}_{\mathrm{n}+\mathrm{p}}, \mathrm{t}\right) \geq & \lim _{n \rightarrow \infty} \mathrm{M}\left(\mathrm{v}_{\mathrm{n}}, \mathrm{v}_{\mathrm{n}+1}, \frac{t}{p}\right) * \ldots * \\
& \lim _{n \rightarrow \infty} \mathrm{M}\left(\mathrm{v}_{\mathrm{n}+\mathrm{p}-1}, \mathrm{v}_{\mathrm{n}+\mathrm{p}}, \frac{t}{p}\right) \\
\geq & 1 * \ldots * 1 \\
& =1 .
\end{aligned}
$$

That is, $\lim _{n \rightarrow \infty} \mathrm{M}\left(\mathrm{v}_{\mathrm{n}}, \mathrm{v}_{\mathrm{n}+\mathrm{p}}, \mathrm{t}\right)=1$.

Hence, $\left\{\mathrm{v}_{\mathrm{n}}\right\}$ is a Cauchy sequence in $\mathrm{X}$.

Since $(X, M, *)$ is a complete fuzzy metric space, there exists $w \in X$ such that $\lim _{n \rightarrow \infty} M\left(v_{n}, w, t\right)=1$, for each $t>0$.

Since $\mathrm{Au}_{2 \mathrm{n}}=\mathrm{Tu}_{2 \mathrm{n}+1}=\mathrm{v}_{2 \mathrm{n}}$ and $\mathrm{Bu}_{2 \mathrm{n}+1}=\mathrm{Su}_{2 \mathrm{n}+2}=\mathrm{v}_{2 \mathrm{n}+1}$ are subsequences of $\left\{\mathrm{v}_{\mathrm{n}}\right\}$,

$\lim _{n \rightarrow \infty} \mathrm{Au}_{2 \mathrm{n}}=\lim _{n \rightarrow \infty} \mathrm{Tu}_{2 \mathrm{n}+1}=\lim _{n \rightarrow \infty} \mathrm{Bu}_{2 \mathrm{n}+1}=\lim _{n \rightarrow \infty} \mathrm{Su}_{2 \mathrm{n}+2}=\mathrm{w}$.

Case 1.Suppose $\mathrm{A}$ is continuous, since $\mathrm{A}$ and $\mathrm{S}$ are compatible and by Lemma 2.7, $\mathrm{AAu}_{2 \mathrm{n}}$ and $\mathrm{SAu}_{2 \mathrm{n}}$ converges to Aw as

$\mathrm{n} \rightarrow \infty$.

Consider,

$\mathrm{M}\left(\mathrm{AAu}_{2 \mathrm{n}}, \mathrm{Bu}_{2 \mathrm{n}+1}, \mathrm{t}\right) \geq \psi\left[\min \left\{\mathrm{M}\left(\mathrm{SAu}_{2 \mathrm{n}}, \mathrm{Tu}_{2 \mathrm{n}+1}, \mathrm{t}\right)\right.\right.$, $\mathrm{M}\left(\mathrm{AAu}_{2 \mathrm{n}}, \mathrm{SAu}_{2 \mathrm{n}}, \mathrm{t}\right)$,

$\mathrm{M}\left(\mathrm{Bu}_{2 \mathrm{n}+1}, \mathrm{Tu}_{2 \mathrm{n}+1}, \mathrm{t}\right)$,

$\left.\left.\mathrm{M}\left(\mathrm{SAu}_{2 \mathrm{n}}, \mathrm{Bu}_{2 \mathrm{n}+1}, 2 \mathrm{t}\right), \mathrm{M}\left(\mathrm{AAu}_{2 \mathrm{n}}, \mathrm{Tu}_{2 \mathrm{n}+1}, \mathrm{t}\right)\right\}\right]$

Taking limit as $\mathrm{n} \rightarrow \infty$, we get

$\mathrm{M}(\mathrm{Aw}, \mathrm{w}, \mathrm{t}) \geq \psi[\min \{\mathrm{M}(\mathrm{Aw}, \mathrm{w}, \mathrm{t}), \mathrm{M}(\mathrm{A} w, A w, t), \mathrm{M}(\mathrm{w}, \mathrm{w}, \mathrm{t})$, $M(A w, w, 2 t), M(A w, w, t)\}]$

$=\psi[\min \{\mathrm{M}(\mathrm{Aw}, \mathrm{w}, \mathrm{t}), \mathrm{M}(\mathrm{Aw}, \mathrm{w}, 2 \mathrm{t}), 1\}]$

$=\psi(\mathrm{M}(\mathrm{Aw}, \mathrm{w}, \mathrm{t}))$.

That is, $M(A w, w, t) \geq \psi(M(A w, w, t))$.

Since $\psi(s) \geq s$ for all $s \in(0,1]$, it is possible only when $\mathrm{M}(\mathrm{Aw}, \mathrm{w}, \mathrm{t})=1$.

That is, Aw $=w$.

Since $\mathrm{AX} \subset \mathrm{TX}$ and hence there exists a point $\mathrm{x} \in \mathrm{X}$ such that $\mathrm{w}=\mathrm{Aw}=\mathrm{Tx}$.

We claim that $\mathrm{w}=\mathrm{Bx}$.

$\mathrm{M}\left(\mathrm{AAu}_{2 \mathrm{n}}, \mathrm{Bx}, \mathrm{t}\right) \geq \psi\left[\min \left\{\mathrm{M}\left(\mathrm{SAu}_{2 \mathrm{n}}, \mathrm{Tx}, \mathrm{t}\right)\right.\right.$, $\mathrm{M}\left(\mathrm{AAu}_{2 \mathrm{n}}, \mathrm{SAu}_{2 \mathrm{n}}, \mathrm{t}\right), \mathrm{M}(\mathrm{Bx}, \mathrm{Tx}, \mathrm{t})$, $\left.\left.\mathrm{M}\left(\mathrm{SAu}_{2 \mathrm{n}}, \mathrm{Bx}, 2 \mathrm{t}\right), \mathrm{M}\left(\mathrm{AAu}_{2 \mathrm{n}}, \mathrm{Tx}, \mathrm{t}\right)\right\}\right]$.

Takin limit as $\mathrm{n} \rightarrow \infty$, we get

$\mathrm{M}(\mathrm{Aw}, \mathrm{Bx}, \mathrm{t})$

$\psi[\min \{\mathrm{M}(\mathrm{Aw}, \mathrm{Aw}, \mathrm{t}), \mathrm{M}(\mathrm{Aw}, \mathrm{Aw}, \mathrm{t}), \mathrm{M}(\mathrm{Bx}, \mathrm{Aw}, \mathrm{t})$, $\mathrm{M}(\mathrm{Aw}, \mathrm{Bx}, 2 \mathrm{t}), \mathrm{M}(\mathrm{Aw}, \mathrm{Aw}, \mathrm{t})\}]$

That is, $\quad \mathrm{M}(\mathrm{w}, \mathrm{Bx}, \mathrm{t})$ = $\psi[\min \{1,1, \mathrm{M}(\mathrm{Bx}, \mathrm{w}, \mathrm{t}), \mathrm{M}(\mathrm{w}, \mathrm{Bx}, 2 \mathrm{t}), 1\}]$

$$
=\psi[\mathrm{M}(\mathrm{w}, \mathrm{Bx}, \mathrm{t})]
$$

That is, $M(w, B x, t) \geq \psi[M(w, B x, t)]$.

It is possible only when $M(w, B x, t)=1$. That is, $w=B x$.

Since $\mathrm{B}$ and $\mathrm{T}$ are compatible and $\mathrm{Bx}=\mathrm{Tx}$,

by Proposition 3.1, BTx $=\mathrm{TBx}$ and $\mathrm{Tw}=\mathrm{TBx}=\mathrm{BTx}=$ Bw.

Published By: 
Also we have,

$\mathrm{M}(\mathrm{Au} 2 \mathrm{n}, \mathrm{Bw}, \mathrm{t}) \geq \psi\left[\min \left\{\mathrm{M}\left(\mathrm{Su}_{2 \mathrm{n}}, \mathrm{Tw}, \mathrm{t}\right), \mathrm{M}\left(\mathrm{Au}_{2 \mathrm{n}}, \mathrm{Su}_{2 \mathrm{n}}, \mathrm{t}\right)\right.\right.$, $\mathrm{M}(\mathrm{Bw}, \mathrm{Tw}, \mathrm{t})$,

$\left.\left.\mathrm{M}\left(\mathrm{Su}_{2 \mathrm{n}}, \mathrm{Bw}, 2 \mathrm{t}\right), \mathrm{M}\left(\mathrm{Au}_{2 \mathrm{n}}, \mathrm{Tw}, \mathrm{t}\right)\right\}\right]$.

Taking limit as $\mathrm{n} \rightarrow \infty$, we get

$\mathrm{M}(\mathrm{w}, \mathrm{Bw}, \mathrm{t}) \geq \psi[\min \{\mathrm{M}(\mathrm{w}, \mathrm{Bw}, \mathrm{t}), \quad \mathrm{M}(\mathrm{w}, \mathrm{w}, \mathrm{t}), 1$,

$\mathrm{M}(\mathrm{w}, \mathrm{Bw}, 2 \mathrm{t})$,

$\mathrm{M}(\mathrm{w}, \mathrm{Bw}, \mathrm{t})\}]$

$=\psi[\mathrm{M}(\mathrm{w}, \mathrm{Bw}, \mathrm{t})]$.

That is, $\mathrm{M}(\mathrm{w}, \mathrm{Bw}, \mathrm{t}) \geq \psi[\mathrm{M}(\mathrm{w}, \mathrm{Bw}, \mathrm{t})]$.

It is possible only when $\mathrm{M}(\mathrm{w}, \mathrm{Bw}, \mathrm{t})=1$.

That is $\mathrm{w}=\mathrm{Bw}=\mathrm{Tw}$.

Since $\mathrm{BX} \subset \mathrm{SX}$, there exists a point $\mathrm{y} \in \mathrm{X}$ such that

$\mathrm{w}=\mathrm{Bw}=\mathrm{Sy}$.

We claim that $\mathrm{w}=\mathrm{Ay}$.

$\mathrm{M}(\mathrm{Ay}, \mathrm{w}, \mathrm{t})=\mathrm{M}(\mathrm{Ay}, \mathrm{Bw}, \mathrm{t})$

$\mathrm{M}(\mathrm{Bw}, \mathrm{Tw}, \mathrm{t})$,

$\geq \quad \psi[\min \{\mathrm{M}(\mathrm{Sy}, \mathrm{Tw}, \mathrm{t}), \quad \mathrm{M}(\mathrm{Ay}, \mathrm{Sy}, \mathrm{t})$,

Taking limit as $\mathrm{n} \rightarrow \infty$, we get

$\mathrm{M}(\mathrm{Ay}, \mathrm{w}, \mathrm{t}) \geq \psi[\min \{\mathrm{M}(\mathrm{w}, \mathrm{w}, \mathrm{t}), \mathrm{M}(\mathrm{Ay}, \mathrm{w}, \mathrm{t}), 1, \mathrm{M}(\mathrm{w}, \mathrm{w}, 2 \mathrm{t})$, $\mathrm{M}(\mathrm{Ay}, \mathrm{w}, \mathrm{t})\}]$

$$
\geq \psi[\mathrm{M}(\mathrm{Ay}, \mathrm{w}, \mathrm{t})]
$$$$
=1 \text {. }
$$

Since $\psi(t)>t$ for all $t \in(0,1)$ and $\psi(1)=1, M($ Ay, $w, t)=$ 1. That is, $A y=w$.

Since $\mathrm{A}$ and $\mathrm{S}$ are compatible and $\mathrm{Ay}=\mathrm{Sy}$, by Proposition 3.1, ASy $=$ Say and hence $\mathrm{Sw}=\mathrm{SAy}=\mathrm{ASy}=$ Aw.

Hence, $\mathrm{w}=\mathrm{Bw}=\mathrm{Tw}=\mathrm{Aw}=\mathrm{Sw}$.

Therefore, $\mathrm{w}$ is a common fixed point of A,B,S and T.

Case 2. Similarly, we can prove when B is continuous.

Case 3. Suppose $\mathrm{S}$ is continuous, since $\mathrm{A}$ and $\mathrm{S}$ are compatible and by Lemma 2.7, $\mathrm{SSu}_{2 \mathrm{n}}$ and $\mathrm{ASu}_{2 \mathrm{n}}$ converges to $\mathrm{Sw}$ as

$\mathrm{n} \rightarrow \infty$.

Consider,

$\mathrm{M}\left(\mathrm{ASu}_{2 \mathrm{n}}, \mathrm{Bu}_{2 \mathrm{n}+1}, \mathrm{t}\right) \geq \psi\left[\min \left\{\mathrm{M}\left(\mathrm{SSu}_{2 \mathrm{n}}, \mathrm{Tu}_{2 \mathrm{n}+1}, \mathrm{t}\right)\right.\right.$, $\mathrm{M}\left(\mathrm{ASu}_{2 \mathrm{n}}, \mathrm{SSu}_{2 \mathrm{n}}, \mathrm{t}\right)$,

$\mathrm{M}\left(\mathrm{Bu}_{2 \mathrm{n}+1}, \mathrm{Tu}_{2 \mathrm{n}+1}, \mathrm{t}\right)$,

$\left.\left.\mathrm{M}\left(\mathrm{SSu}_{2 \mathrm{n}}, \mathrm{Bu}_{2 \mathrm{n}+1}, 2 \mathrm{t}\right), \mathrm{M}\left(\mathrm{ASu}_{2 \mathrm{n}}, \mathrm{Tu}_{2 \mathrm{n}+1}, \mathrm{t}\right)\right\}\right]$

Taking limit as $\mathrm{n} \rightarrow \infty$, we get

$\mathrm{M}(\mathrm{Sw}, \mathrm{w}, \mathrm{t}) \geq \psi[\min \{\mathrm{M}(\mathrm{Sw}, \mathrm{w}, \mathrm{t}), \mathrm{M}(\mathrm{Sw}, \mathrm{Sw}, \mathrm{t}), \mathrm{M}(\mathrm{w}, \mathrm{w}, \mathrm{t})$,

$\mathrm{M}(\mathrm{Sw}, \mathrm{w}, 2 \mathrm{t}), \mathrm{M}(\mathrm{Sw}, \mathrm{w}, \mathrm{t})\}]$

$=\psi[\min \{\mathrm{M}(\mathrm{Sw}, \mathrm{w}, \mathrm{t}), \mathrm{M}(\mathrm{Sw}, \mathrm{w}, 2 \mathrm{t}), 1\}]$

$=\psi(\mathrm{M}(\mathrm{Sw}, \mathrm{w}, \mathrm{t}))$.

That is, $\mathrm{M}(\mathrm{Sw}, \mathrm{w}, \mathrm{t}) \geq \psi(\mathrm{M}(\mathrm{Sw}, \mathrm{w}, \mathrm{t}))$.

Since $\psi(s) \geq s$ for all $s \in(0,1]$, it is possible only when

$\mathrm{M}(\mathrm{Sw}, \mathrm{w}, \mathrm{t})=1$. That is, $\mathrm{Sw}=\mathrm{w}$.

Now, we claim that $w=A w$.

$\mathrm{M}\left(\mathrm{Aw}, \mathrm{Bu}_{2 \mathrm{n}+1}, \mathrm{t}\right) \geq \psi\left[\min \left\{\mathrm{M}\left(\mathrm{Sw}, \mathrm{Tu}_{2 \mathrm{n}+1}, \mathrm{t}\right), \mathrm{M}(\mathrm{Aw}, \mathrm{Sw}, \mathrm{t})\right.\right.$,

$$
\mathrm{M}\left(\mathrm{Bu}_{2 \mathrm{n}+} 1, \mathrm{Tu}_{2 \mathrm{n}+1}, \mathrm{t}\right), \mathrm{M}\left(\mathrm{Sw}, \mathrm{Bu}_{2 \mathrm{n}+} 1,2 \mathrm{t}\right) \text {, }
$$$$
\left.\left.\mathrm{M}\left(\mathrm{Aw}, \mathrm{Tu}_{2 \mathrm{n}+1}, \mathrm{t}\right)\right\}\right]
$$

Taking limit as $\mathrm{n} \rightarrow \infty$, we get

$\mathrm{M}(\mathrm{Aw}, \mathrm{w}, \mathrm{t}) \geq \psi[\min \{\mathrm{M}(\mathrm{w}, \mathrm{w}, \mathrm{t}), \mathrm{M}(\mathrm{w}, \mathrm{w}, \mathrm{t}), \mathrm{M}(\mathrm{w}, \mathrm{w}, \mathrm{t})$, $M(w, w, 2 t), M(w, w, t)\}]$

$$
\begin{aligned}
& \geq \psi[1] \\
& =1 .
\end{aligned}
$$

Since $\psi(t)>t$ for all $t \in(0,1)$ and $\psi(1)=1, M(w, A w, t)=$ 1. That is, Aw $=w$.
Since AX $\subset \mathrm{TX}$ and hence there exists a point $\mathrm{z} \in \mathrm{X}$ such that $\mathrm{w}=\mathrm{Aw}=\mathrm{Tz}$.

We claim that $\mathrm{w}=\mathrm{Bz}$.

$\mathrm{M}(\mathrm{w}, \mathrm{Bz}, \mathrm{t})=\mathrm{M}(\mathrm{Aw}, \mathrm{Bz}, \mathrm{t})$

$\geq \quad \psi[\min \{\mathrm{M}(\mathrm{Sw}, \mathrm{Tz}, \mathrm{t}), \quad \mathrm{M}(\mathrm{Aw}, \mathrm{Sw}, \mathrm{t})$,

$\mathrm{M}(\mathrm{Bz}, \mathrm{Tz}, \mathrm{t})$,

$\mathrm{M}(\mathrm{Sw}, \mathrm{Bz}, 2 \mathrm{t}), \mathrm{M}(\mathrm{Aw}, \mathrm{Tz}, \mathrm{t})\}]$.

$\geq \psi[\min \{\mathrm{M}(\mathrm{w}, \mathrm{w}, \mathrm{t}), \mathrm{M}(\mathrm{w}, \mathrm{w}, \mathrm{t}), \mathrm{M}(\mathrm{Bz}, \mathrm{w}, \mathrm{t})$, $\mathrm{M}(\mathrm{w}, \mathrm{Bz}, 2 \mathrm{t}), 1\}]$.

$=\psi[\mathrm{M}(\mathrm{w}, \mathrm{Bz}, \mathrm{t})]$.

That is, $\mathrm{M}(\mathrm{w}, \mathrm{Bz}, \mathrm{t}) \geq \psi[\mathrm{M}(\mathrm{w}, \mathrm{Bz}, \mathrm{t})]$.

It is possible only when $\mathrm{M}(\mathrm{w}, \mathrm{Bz}, \mathrm{t})=1$. That is, $\mathrm{w}=\mathrm{Bz}$.

Since $\mathrm{B}$ and $\mathrm{T}$ are compatible and $\mathrm{Bz}=\mathrm{Tz}$, by Proposition 3.1, $\mathrm{BTz}=\mathrm{TBz}$ and $\mathrm{Tw}=\mathrm{TBz}=\mathrm{BTz}=\mathrm{Bw}$.

Also, we claim that $\mathrm{w}=\mathrm{Bw}$.

$\mathrm{M}(\mathrm{w}, \mathrm{Bw}, \mathrm{t})=\mathrm{M}(\mathrm{Aw}, \mathrm{Bw}, \mathrm{t})$

$\mathrm{M}(\mathrm{Bw}, \mathrm{Tw}, \mathrm{t})$

$$
\geq \quad \psi[\min \{\mathrm{M}(\mathrm{Sw}, \mathrm{Tw}, \mathrm{t}), \quad \mathrm{M}(\mathrm{Aw}, \mathrm{Sw}, \mathrm{t}),
$$

$\mathrm{M}(\mathrm{Sw}, \mathrm{Bw}, 2 \mathrm{t}), \mathrm{M}(\mathrm{Aw}, \mathrm{Tw}, \mathrm{t})\}]$.

$\geq \psi[\min \{\mathrm{M}(\mathrm{w}, \mathrm{Bw}, \mathrm{t}), \mathrm{M}(\mathrm{w}, \mathrm{w}, \mathrm{t}), 1, \mathrm{M}(\mathrm{w}, \mathrm{Bw}, 2 \mathrm{t})$, $\mathrm{M}(\mathrm{w}, \mathrm{Bw}, \mathrm{t})\}]$

$=\psi[\mathrm{M}(\mathrm{w}, \mathrm{Bw}, \mathrm{t})]$

That is, $\mathrm{M}(\mathrm{w}, \mathrm{Bw}, \mathrm{t}) \geq \psi[\mathrm{M}(\mathrm{w}, \mathrm{Bw}, \mathrm{t})]$.

It is possible only when $\mathrm{M}(\mathrm{w}, \mathrm{Bw}, \mathrm{t})=1$. That is, $\mathrm{w}=\mathrm{Bw}$.

Hence $\mathrm{w}=\mathrm{Aw}=\mathrm{Sw}=\mathrm{Bw}=\mathrm{Tw}$. Therefore, $\mathrm{w}$ is a common fixed point of A,B,S and $\mathrm{T}$.

Case 4. Similarly, we can prove when $\mathrm{T}$ is continuous.

\section{Uniqueness:}

Suppose $\mathrm{w}_{1}$ is also a common fixed point of A,B,S and T.

$\mathrm{M}\left(\mathrm{w}, \mathrm{w}_{1}, \mathrm{t}\right)=\mathrm{M}\left(\mathrm{Aw}, \mathrm{Bw}_{1}, \mathrm{t}\right)$

$\geq \psi\left[\min \left\{\mathrm{M}\left(\mathrm{Sw}, \mathrm{Tw}_{1}, \mathrm{t}\right), \mathrm{M}(\mathrm{Aw}, \mathrm{Sw}, \mathrm{t})\right.\right.$,

$$
\mathrm{M}\left(\mathrm{Bw}_{1}, \mathrm{Tw}_{1}, \mathrm{t}\right)
$$

$\left.\left.\mathrm{M}\left(\mathrm{Sw}, \mathrm{Bw}_{1}, 2 \mathrm{t}\right), \mathrm{M}\left(\mathrm{Aw}, \mathrm{Tw}_{1}, \mathrm{t}\right)\right\}\right]$

$=\psi\left[\min \left\{\mathrm{M}\left(\mathrm{w}, \mathrm{w}_{1}, \mathrm{t}\right), \mathrm{M}(\mathrm{w}, \mathrm{w}, \mathrm{t}), \mathrm{M}\left(\mathrm{w}_{1}, \mathrm{w}_{1}, \mathrm{t}\right)\right.\right.$, $\left.\left.M\left(w, w_{1}, 2 t\right), M\left(w, w_{1}, t\right)\right\}\right]$

$=\psi\left[\mathrm{M}\left(\mathrm{w}, \mathrm{w}_{1}, \mathrm{t}\right)\right]$.

That is, $\mathrm{M}\left(\mathrm{w}, \mathrm{w}_{1}, \mathrm{t}\right) \geq \psi\left[\mathrm{M}\left(\mathrm{w}, \mathrm{w}_{1}, \mathrm{t}\right)\right]$

This is possible only when $\mathrm{M}\left(\mathrm{w}, \mathrm{w}_{1}, \mathrm{t}\right)=1$. That is, $\mathrm{w}=$ $\mathrm{w}_{1}$. Hence the proof.

Example 3.3. Let $\mathrm{X}=(-\infty, \infty)$ with the metric $\mathrm{d}$ defined by $\mathrm{d}(\mathrm{u}, \mathrm{v})=|\mathrm{u}-\mathrm{v}|$, define $\mathrm{M}(\mathrm{u}, \mathrm{v}, \mathrm{t})=\frac{t}{t+d(u, v)}$, for all $\mathrm{u}, \mathrm{v} \in$ $\mathrm{X}$ and $\mathrm{t}>0$. Note that, $(\mathrm{X}, \mathrm{M}, *)$ where $\mathrm{a} * \mathrm{~b}=\min (\mathrm{a}, \mathrm{b})$ is $\mathrm{a}$ complete fuzzy metric space.

The maps A,B,S,T $: X \rightarrow X$ is defined by

$\mathrm{A}(\mathrm{u})=\frac{2+u}{3}, \mathrm{~B}(\mathrm{u})=3-2 \mathrm{u}, \mathrm{S}(\mathrm{u})=2-\mathrm{u}$ and $\mathrm{T}(\mathrm{u})=\mathrm{u}$.

Let $\mathrm{u}_{\mathrm{n}}=\left(1-\frac{1}{n}\right)$.

Now, we verify that A,S is compatible. 


\section{GENERALIZATION OF SINGH'S COMMON FIXED POINT THEOREM}

$$
\begin{aligned}
\lim _{n \rightarrow \infty} \mathrm{M}\left(\mathrm{ASu}_{\mathrm{n}}, \mathrm{SAu}_{\mathrm{n}}, \mathrm{t}\right) & =\lim _{n \rightarrow \infty} \mathrm{M}\left(\mathrm{A} 2-\mathrm{u}_{\mathrm{n}}, \mathrm{S} 2+\mathrm{u}_{\mathrm{n}} 3, \mathrm{t}\right) \\
& =\lim _{n \rightarrow \infty} \mathrm{M}\left(\frac{4-u_{n}}{3}, \frac{4-u_{n}}{3}, \mathrm{t}\right) \\
& =1 .
\end{aligned}
$$

$\lim _{n \rightarrow \infty} \mathrm{M}\left(\mathrm{ASu}_{\mathrm{n}}, \mathrm{SAu}_{\mathrm{n}}, \mathrm{t}\right)=1$

Also $\lim _{n \rightarrow \infty} \mathrm{Au}_{\mathrm{n}}=\lim _{n \rightarrow \infty} \frac{2+u_{n}}{3}=\lim _{n \rightarrow \infty} \frac{2+\left(1-\frac{1}{n}\right)}{3}=1$.

$\lim _{n \rightarrow \infty} \mathrm{Su}_{\mathrm{n}}=\lim _{n \rightarrow \infty} 2-\mathrm{u}_{\mathrm{n}}=\lim _{n \rightarrow \infty} 2-\left(1-\frac{1}{n}\right)=1$.

Therefore, $\mathrm{A}$ and $\mathrm{S}$ are compatible mapping.

Similarly, we can verify that $\mathrm{B}$ and $\mathrm{T}$ are compatible. Also

$\mathrm{BX} \subset \mathrm{SX}$ and $\mathrm{AX} \subset \mathrm{TX}$ and $\mathrm{T}$ is continuous.

Define the map $\psi:(0,1] \rightarrow(0,1]$ by $\psi(\mathrm{s})=\frac{2 s}{s+1}$ for each $\mathrm{s} \in(0,1]$. Now, we verify that

$\mathrm{M}(\mathrm{Au}, \mathrm{Bv}, \mathrm{t}) \geq \psi[\min \{\mathrm{M}(\mathrm{Su}, \mathrm{Tv}, \mathrm{t}), \mathrm{M}(\mathrm{Au}, \mathrm{Su}, \mathrm{t}), \mathrm{M}(\mathrm{Bv}, \mathrm{Tv}, \mathrm{t})$, $\mathrm{M}(\mathrm{Su}, \mathrm{Bv}, 2 \mathrm{t}), \mathrm{M}(\mathrm{Au}, \mathrm{Tv}, \mathrm{t})\}]$, for all $\mathrm{u}, \mathrm{v}$

$\in \mathrm{X}$.

Consider the following:

Suppose $\mathrm{u}=4$ and $\mathrm{v}=10$.

$\mathrm{M}(\mathrm{A} 4, \mathrm{~B} 10, \mathrm{t}) \geq \psi[\min \{\mathrm{M}(\mathrm{S} 4, \mathrm{~T} 10, \mathrm{t}), \mathrm{M}(\mathrm{A} 4, \mathrm{~S} 4, \mathrm{t})$, $\mathrm{M}(\mathrm{B} 10, \mathrm{~T} 10, \mathrm{t}), \mathrm{M}(\mathrm{S} 4, \mathrm{~B} 10,2 \mathrm{t})$,

$\mathrm{M}(\mathrm{A} 4, \mathrm{~T} 10, \mathrm{t})\}]$

if $\quad \mathrm{M}(2,-17, \mathrm{t}) \quad \geq \quad \psi[\min \{\mathrm{M}(-2,10, \mathrm{t})$,

$\mathrm{M}(2,-2, \mathrm{t}), \mathrm{M}(-17,10, \mathrm{t})$,

$$
\mathrm{M}(-2,-17,2 \mathrm{t}), \mathrm{M}(2,10, \mathrm{t})\}]
$$

That is if $\frac{t}{t+15} \geq \psi\left[\min \left\{\frac{t}{t+12}, \frac{t}{t+4}, \frac{t}{t+27}\right.\right.$,

$$
\left.\left.\frac{t}{t+7.5}, \frac{t}{t+15}\right\}\right]
$$

That is if $\frac{t}{t+15} \geq \psi\left[\frac{t}{t+27}\right]$

That is if $\frac{t}{t+15} \geq \frac{t}{t+13.5}$

That is if $15 \geq 13.5$.

Similarly, we can verify, for all $u, v \in(-\infty, \infty)$.

All the conditions of the previous theorem are verified.

Then, 1 is the unique fixed point.

Hence, $\mathrm{A}, \mathrm{B}, \mathrm{S}$ and $\mathrm{T}$ have the common fixed point in $\mathrm{X}$.

\section{REFERENCES}

1. Bijendra Singh and M.S. Chauhan, "Common fixed points of Compatible maps in fuzzy metric spaces", Fuzzy sets and systems 115(3)(2000), 471-475.

2. A. George and P. Veeramani, "On some results in fuzzy metric spaces", Fuzzy sets and systems 64(3)(1994), 395-399.

3. Gerald Jungck, "Compatible mappings and common fixed points", International journal of Mathematics and Mathematical sciences 9(4)(1986), 771-779.

4. M. Grabiec, "Fixed points in fuzzy metric spaces", Fuzzy sets and Systems 27(3)(1988), 385-389.

5. Ivan Kramsoil and Jiri Michalek, "Fuzzy metrics and statistical metric Spaces", Kybernetica 11(5)(1975), 336-344.
6. J. Jeyachristy Priskillal and P. Thangavelu, "Intuitionistic fuzzy $(\psi, \eta)$-contractive mapping and fixed points", Journal of Analysis and Number Theory 5(2)(2017), 1-4.

7. S.N. Mishra, Nilima Sharma and S.L Singh, "Common fixed points of maps on fuzzy metric spaces", International journal of Mathematics and Mathematical sciences 17(2)(1994), 253258.

8. R.P. Pant, "Common fixed points of noncomputing mappings", Journal of Mathematical analysis and applications 188(2)(1994), 436-440.

9. B. Schweizer and A. Sklar, "Statistical metric spaces", Pacific journal of Mathematics 10(1)(1960), 385-389.

10. K.P.R. Sedghi, Rao and N. Shobe, "A common fixed point theorem for six weakly compatible maps in M-fuzzy metric space"s, Iranian journal of fuzzy systems 5(2)(2008), 49-62.

11. G. Song, "Comments on: a common fixed point theorem in a fuzzy metric space”, Fuzzy sets and systems 135(2003), 409413.

12. Sushil Sharma, Common fixed point theorems in fuzzy metric spaces, Fuzzy sets and systems 127(3)(2002), 345-352.

13. R. Vasuki, Common fixed points for R-weakly commuting maps in fuzzy metric spaces, Indian journal of pure and applied Mathematics 30(4)(1999), 419-423. 